\title{
Time-Delay Neural Network for Smart MIMO Channel Estimation in Downlink 4G-LTE- Advance System
}

\author{
Nirmalkumar S. Reshamwala \\ Electronics and Communication Engineering Department, Sarvajanik College of Engineering and Technology (SCET), \\ Surat, Gujarat, India \\ Email: reshamwala.nirmal01@gmail.com \\ Pooja S. Suratia \\ Department of Electrical Engineering, The Maharaja Sayajirao University of Baroda, Vadodara, Gujarat, India \\ Email: poojasuratia@yahoo.com \\ Satish K. Shah \\ Department of Electrical Engineering, The Maharaja Sayajirao University of Baroda, Vadodara, Gujarat, India \\ Email: satishkshah_2005@yahoo.com3
}

\begin{abstract}
Long-Term Evolution (LTE) is the next generation of current mobile telecommunication networks. LTE has a new flat radio-network architecture and significant increase in spectrum efficiency. In this paper, main focus on throughput performance analysis of robust MIMO channel estimators for Downlink Long Term Evolution-Advance (DL LTE-A)-4G system using three Artificial Neural Networks: Feed-forward neural network (FFNN), Cascade-forward neural network (CFNN) and Time-Delay neural network (TDNN) are adopted to train the constructed neural networks' models separately using Back-Propagation Algorithm. The methods use the information received by the received reference symbols to estimate the total frequency response of the channel in two important phases. In the first phase, the proposed ANN based method learns to adapt to the channel variations, and in the second phase, it estimates the MIMO channel matrix and try to improve throughput of LTE. The performance of the estimation methods is evaluated by simulations in Vienna LTE-A DL Link Level Simulator. Performance of the proposed channel estimator, Time-Delay neural network (TDNN) is compared with traditional Least Square (LS) algorithm and ANN based other estimators for Closed Loop Spatial Multiplexing (CLSM) - Single User Multi-input Multi-output (MIMO-2×2 and 4×4) in terms of throughput. Simulation result shows TDNN gives better performance than other ANN based estimations methods and LS.
\end{abstract}

Index Terms-LTE-A, OFDM-MIMO, Back-Propagation, Feed-forward neural network (FFNN), Cascade-forward neural network (CFNN), Time-Delay neural network (TDNN)

\section{INTRODUCTION}

Research beyond $3 \mathrm{G}$ mobile radio systems is in progress around the world to allow future mobile networks to support different types of services and applications with high performance as a natural evolution of GSM (Global system for mobile communications) and UMTS (Universal Mobile Telecommunications System).
The advances in mobile device technologies, together with the accessibility provided by those devices to the Internet and the numerous applications and services that come with it, are central to need for this research [1]. The 3rd Generation Partnership Group standardized Evolved (E-UTRA) as Long Term Evolution to be used as a Next Generation Wireless Network. It is a step towards the fourth generation-4G (LTE-A) that is being developed by 3rd Generation Partnership Project (3GPP), a new standard as the evolution of the current network architecture of mobile communications, GSM/HSPA to increase maximum user capacity, the spectral efficiency, low latency and to obtain higher throughput [2-3]. LTE towards LTE-Advanced- $4 \mathrm{G}$ is set to provide higher bit rates in a cost efficient way and, at the same time, completely fulfill the requirements set by International Telecommunication Union (ITU) for International Mobile Telecommunications-Advanced (IMT Advanced) also referred to as $4 \mathrm{G}$. The features supported by LTE-A are given in [4]. Release 10 support enhanced MIMO Techniques with $8 \mathrm{X} 8$ in downlink and upto $4 \mathrm{X} 4$ in uplink with wider bandwidths, enabled by carrier aggregation. It achieves maximum Peak data rate $1 \mathrm{Gbps}$ for downlink and $500 \mathrm{Mbps}$ for uplink. LTE-Advanced downlink uses an Orthogonal Frequency Division Multiplex Access (OFDMA) radio interface in downlink and the SingleCarrier Frequency Division Multiple Access (SC-FDMA) for the uplink [5-6].

The receiver in OFDM-MIMO system requires the knowledge of Channel State Information (CSI) with view to recovering the original transmitted signal data properly without noise. In certain channel estimation methods, pilot symbols are inserted and transmitted over the channel, and are estimated at the receiver in order to recover original transmitted symbols [6-7]. The most traditional efficient training based methods are the Least 
Squares (LS), Minimum Mean Square Error (MMSE) method and Adaptive Filtering channel estimation method are given in [8-9]. In this paper, Channel estimation by artificial neural networks has been deployed in LTE-Advance system, with three different neural networks. In this paper contribution, we propose Study of Different Neural Networks on Throughput Performance of MIMO Channel Estimation for Downlink LTE-Advance System is presented. The principle of this method is to exploit the information provided by the received reference symbols to estimate the channel response using channel matrix estimated by conventional LS Estimator [6].

This work is organized as follows. In section II, the LTE-A Downlink Physical Layer and The Vienna LTE-A Link Level Simulator is described. Section III presents different channel estimation techniques like Least Square (LS) and ANN based techniques. Simulation results and throughput performance analysis of proposed ANN based channel estimation techniques are provided in Section IV. Finally, conclusion and future work is discussed in Section V.

\section{LTE-A DOWNLINK PHYSICAL LAYER AND VIENNA LTE-A LINK LEVEL SIMULATOR}

\subsection{LTE-A Downlink Physical Layer}

Various models LTE-advance Physical Layer is highly efficient means of conveying both data and control information between an enhanced base station (called eNodeB in LTE terminology) and mobile user equipment (UE). Although, LTE physical layer specification describes both FDD (Frequency Division Duplex) and TDD (Time Division Duplex), the study in this paper is focused on Frequency Division Duplex, thus only LTE physical layer with FDD is discussed below. Furthermore, only downlink data transmission is considered for channel estimation. OFDMA-MIMO system is described in $[7,10]$. The orthogonal frequency subcarriers are used to share spectrum among users using access technique. The LTE-A Physical layer employs advanced technologies of wireless cellular systems.

LTE-A Downlink Physical Layer [11-12] is described as shown in Fig. 1. Physical channel processing at eNodeB consists of Scrambling which breaks long strings of $1 \mathrm{~s}$ and 0 s into scrambled bits, Modulation converts scrambled bits into complex-valued symbols uses either QPSK, 16-QAM or 64-QAM modulation, Layer mapper and precoder performs symbol transformations to proceed MIMO transmission techniques, Resource element (RE) mapper maps the symbols to proper locations in the timefrequency Resource grid in physical resource block, OFDM signal mapper generates time domain baseband signals for each antenna port depending upon port $0,1,2$ or 4 for transmission [11]. Similarly, at receiver side descrambling, demodulation and demapper operations and MIMO Receiver processing to recover the transmitted data streams. LTE-A Downlink modulation is based on OFDMA which provides multi user access, robustness to time dispersion of radio channel, and low complexity for receiver design. Also, OFDMmulticarrier concept enables the operation of LTE in various system bandwidths up to $20 \mathrm{MHz}$ by adapting the number of subcarriers used. In Closed Loop Spatial Multiplexing-CLSM, The uplink feedback values Channel Quality Indicator (CQI), Rank Indicator (RI) and Precoding Matrix Indicator (PMI) are calculated at the receiver, and are feedback to the eNodeB. In CLSM, Each transmit antenna transmits a different data stream. This technique significantly increases the peak data rate over the radio link with data quality [13-14].

LTE Downlink Physical channels convey information from higher layers in the LTE stack. Each physical channel defines algorithms for bit scrambling, modulation, layer mapping, CDD (Cyclic Delay Diversity), precoding and resource element assignment. There are three downlink physical channels in LTE [11].

In LTE Physical Layer, transport channels act as service access point (SAPs) for higher layers. There are four downlink transport channels in LTE system. Finally, Mapping Downlink Physical Channel to Downlink Transport Channel is given in [11].

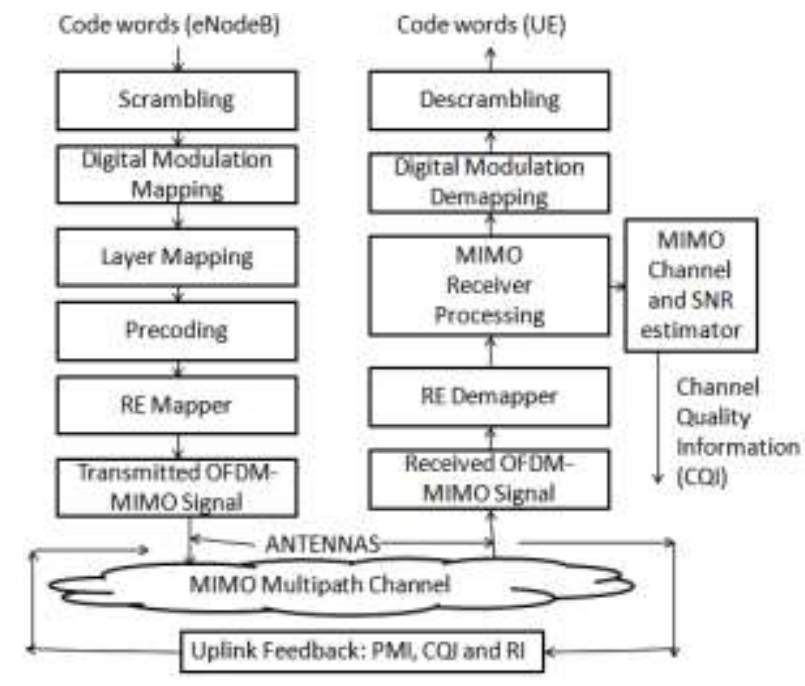

Fig. 1. LTE-A Downlink Physical Layer [6]

\subsection{Vienna LTE-A Link Level Simulator}

Vienna Link Level Simulator [15-16] is used to emulate the transmission of user data and control information from an eNodeB transmitter to a UE receiver modeling the physical layer with high precision. The Link level parameters for the presented simulations are as shown in Table I. Transmission modes in the first release of LTE according to [17] are configured in this LTE-A downLink (DL) Link Level Simulator. Simulated MIMO scheme followed CLSM (Close-Loop Spatial Multiplexing) transmission modes are specified in [17-19].

The MIMO-OFDM physical channel link level simulator emulates the MIMO-OFDM transmission/ reception through a mobile radio channel. The MIMO channel model and reference scenarios employed as reference for the DL link level simulator are described in [20-21]. 


\section{CHANNEL ESTIMATION TECHNIQUES BASED ON LS ESTIMATOR AND ANN}

\subsection{Least Square Channel Estimation}

Least square channel estimator is obtained by minimizing the square distance between the received signal $\bar{Y}$ and the transmitted signal $\underline{X}$ as follows $[6-7,10]$

$$
\begin{aligned}
& \min _{H^{T}} J(H)=\min _{H^{T}}\left\{|\bar{Y}-\underline{X} \bar{H}|^{2}\right\} \\
& =\min _{H^{T}}\left\{(\bar{Y}-\underline{X} \bar{H})^{T}(\bar{Y}-\underline{X} \bar{H})\right\}
\end{aligned}
$$

where, $(.)^{T}$ is the conjugate transpose operator

By differentiating expression (1) with respect to $\bar{H}^{T}$ and finding the minima, we obtain [7]

$$
\frac{\partial}{\partial \bar{H}^{T}} J(H)=-\underline{X}^{T} \bar{Y}+\underline{X}^{T} \underline{X} \bar{H}=0
$$

Finally, the LS channel estimation is given by [6-7,10]

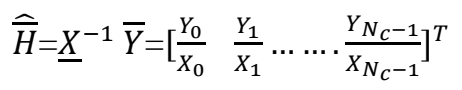

In general, LS channel estimation technique for OFDM systems has low complexity but it suffers from a high mean square error (MSE) [10].

\subsection{Artificial Neural Network}

Neural networks are algorithms for optimization and learning based loosely on concepts inspired by research into the nature of the brain. An artificial neural network is defined as follows: It receives a number of inputs either from original data, or from the output of other neurons with delay or without delay from that ANN or others. Each input goes via a connection that has some strength (weight); these weights correspond to synaptic efficacy in a biological neuron. Each neuron also has a single threshold value. The weighted sum of the inputs is formed, and the threshold subtracted, to compose the activation of the neuron $[6,22]$.

The activation signal is passed through an activation function (also known as a transfer function) to produce the output of the neuron. There can be neurons of hidden layers those play an important role in the neural network. The input, hidden and output neurons need to be connected together [6, 22].

Fig. 2 shows the basic and simple model of feedforward Neural Network with one hidden layer with five hidden neurons with one input and one output.

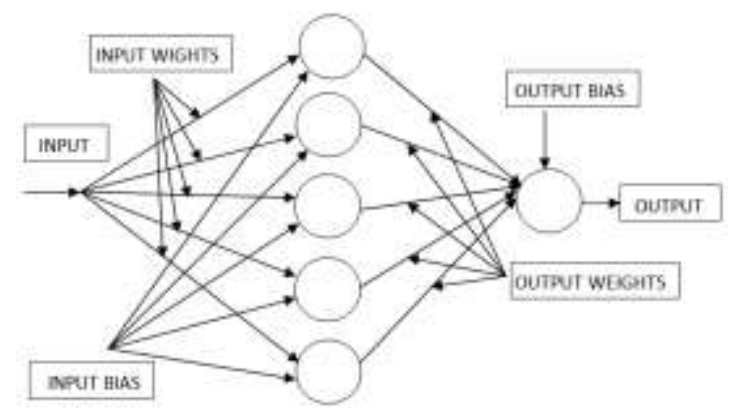

Fig. 2. Simple model of an Artificial Neuron [6]
A simple network has a feed-forward structure; signals flow from inputs, forwards through any hidden units, eventually reaching the output units. Such a structure has stable behaviour. However, if the network is recurrent (contains connections back from later to earlier neurons with unit delay) it can be unstable, and has very complex dynamics [22].

When the network is used in actual application, the input variable values are placed in the input units, then output numerical values of each hidden layers and output layer units are progressively calculated with its activation functions. By taking the weighted sum of the outputs of the units in previous layer, and subtracting the threshold. The activation value is passed through the activation function to produce the output of the neuron. When the entire network has been executed, the outputs of the output layer act as the output of the whole network [6, 22].

A Feed-forward neural Network (FFNN) is one whose topology has no closed paths and number of hidden layer as per requirement and input nodes are connected to the output nodes without any feedback paths. The BackPropagation Algorithm (BPA) uses the steepest-descent method to reach a global minimum with energy function of MSE. The flowchart of the BPA is given in [6].

In Cascade-forward neural network (CFNN), these is similar to feed forward networks such as BackPropagation Neural networks (BPNN) with the exception that they have a weight connection from the input and every previous layer to the following layers [6 ].

Time delay neural network (TDNN) is similar to feedforward networks, except that the input weight has a tap delay line associated with it. This allows the network to have a finite dynamic response to time series input data [23].

\subsection{Channel Estimation using Different ANN}

The simulation program of each one of conducted Back-Propagation training algorithm (Feed-Forward neural network (FFNN), Cascade-Forward neural network (CFNN) and Time delay neural network (TDNN) includes the following steps [6]:

1) First of all, estimate channel using traditional LS estimator using transmitted signal and received signal after passing through Flat Rayleigh and AWGN channel and adding noise as shown in Fig. 3.

2) Initialization of network weights, learning rate and Threshold error. Set all iterations to zero.

3) Take received reference symbols as received signal MIMO matrix as input and estimated MIMO matrix as target

4) Total error = zero; iterations --> iterations +1

5) Feed it to input layer units and Initialize the target output of that ANN.

6) Calculate the outputs of hidden layer units and outputs of output layer units.

7) Calculate the error $=$ desired output - actual output. Total-error --> Total-error + error 
8) Calculate delta sigma of output neurons. Then adjust weights between output and hidden layer units.

9) Calculate delta sigma of hidden layer units. Then adjust weights between hidden and input layer units.

10) While there is more matrix in the file, go to step 4.

11) if Threshold error --> Total_error then stop, otherwise go to step $3^{\text {rd }}$ steps in this algorithm.

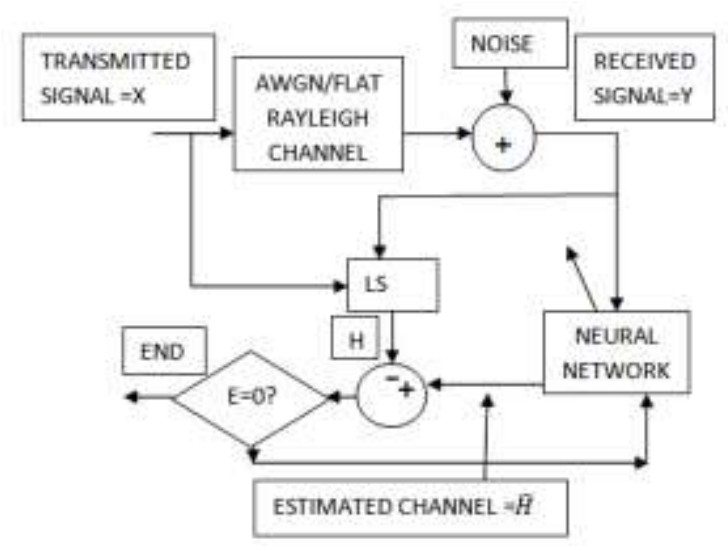

Fig. 3. Channel Estimation using ANN [6]

The estimator uses the information provided by received reference symbols of sub channels to estimate the total channel frequency response. The input of the neural network is the received reference symbols $\mathrm{Y}$, target of ANN is channel estimated by LS $[6,10]$.

\section{Simulation PARAMETERS AND RESUltS}

\subsection{Simulation Parameters for LS Channel Estimator}

The simulation parameters for Vienna LTE-A Link level simulator for LS channel estimator are listed in Table I

Table 1. Simulation Parameters for LS Channel Estimator

\begin{tabular}{|c|c|}
\hline Parameters & Value \\
\hline Number of Sub frames & 25 \\
\hline SNR Range & $\begin{array}{c}{[0,5,10,15,20,25,30]} \\
\text { for AWGN Channel and } \\
{[0,5,10,15,20,25,30,35,40]} \\
\text { for Flat Rayleigh Channel }\end{array}$ \\
\hline Transmission Bandwidth & $1.4 \mathrm{MHz}$ \\
\hline Sub-frame duration & $1 \mathrm{~ms}$ \\
\hline Sub-carrier spacing & $15 \mathrm{kHz}$ \\
\hline FFT size (N) & 128 \\
\hline Number of occupied sub-carriers & 72 \\
\hline TTI length & SU-MIMO ms \\
\hline Simulation Configuration & CLSM-MIMO (2x2 and 4x4) \\
\hline Antenna schemes & ZF (Zero Forcing) \\
\hline MIMO receiver equalizer & AWGN and Flat Rayleigh \\
\hline Channel type & \\
\hline & \\
\hline &
\end{tabular}

\subsection{ANN based simulation parameters}

The simulation parameters for different Neural Networks are as in Table II.

Table 2. Simulation Parameters for FFNN, CFNN and TDNN based Estimators

\begin{tabular}{|c|c|}
\hline \multirow{2}{*}{ Parameters } & FFNN, CFNN AND TDNN \\
\cline { 2 - 2 } & Value \\
\hline Number of inputs & 1 \\
\hline Number of hidden layers & 1 \\
\hline Number of neurons in hidden layer & 5 \\
\hline Epoch number / Iteration & 1000 \\
\hline Training Function / Algorithm & Trainscg \\
\hline Performance & Mean Square Error \\
\hline
\end{tabular}

In Feed-forward neural network (FFNN), Cascadeforward neural network (CFNN) and Time delay neural network (TDNN), the ANN is trained with reference symbols, which is complex type matrix. The target sample set is presented to the ANN in the form of result or estimated complex channel matrix obtained by Least Square (LS). The learning of the ANN is done in the training phase during which the ANN adjusts its weights according to training algorithm. The ANN is trained for 1000 epochs.

In this research paper, Throughput Performance of MIMO Channel Estimation for AWGN and Flat Rayleigh is simulated for downlink LTE-Advanced System using Different Neural Network. Fig. 4, 5, 6, 7, 8, 9, 10 and 11 show the Throughput versus SNR results for comparison of different neural networks based channel estimation method with LS channel estimator and Ideal Channel for Closed Loop Spatial Multiplexing-Single User Multiinput Multi-output $(2 \times 2$ and $4 \times 4)$ (CLSM-SUMIMO).

In FFNN, CFNN and TDNN, the ANN is trained with reference symbols, which is complex type matrix. The target sample set is presented to the ANN in the form of result or estimated channel obtained by Least Square (LS) complex type matrix. The learning of the ANN is done in the training phase during which the ANN adjusts its weights according to training algorithm. The ANN is trained for 1000 epochs.

Fig. 4, 5, 6, and 7 show the Throughput versus SNR results for different ANN based channel estimation method with LS channel estimator and Ideal Channel for Closed Loop Spatial Multiplexing (CLSM)-Single User Multi-input Multi-output (MIMO-2×2 and $4 \times 4$ ) for AWGN Channel in LTE-A simulator. Fig. 5 and 7 are column chart representation of Fig. 4 and 6, respectively.

From Fig. 4, 5, 6, and 7, Cascade-forward neural network (CFNN) is the worst ANN for this application. Feed-forward neural network (FFNN) is giving better performance than CFNN. But, Time delay neural network (TDNN) is the most effective to improve performance and shows better results for SNR range $0 \mathrm{~dB}$ to $30 \mathrm{~dB}$ when compared to ANN based Channel estimators using FFNN and CFNN and traditional method LS estimator. 


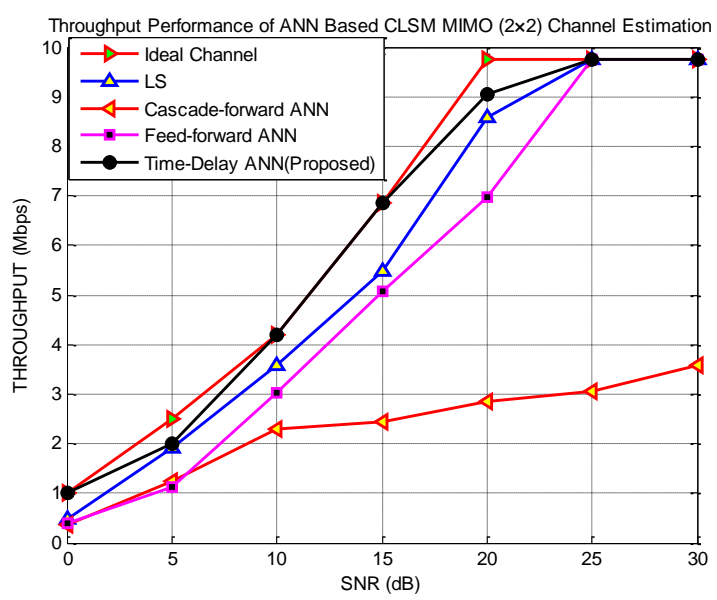

Fig. 4. Throughput v/s SNR performance for ANN based channel estimators in CLSM-MIMO $(2 \times 2)$ for AWGN Channel

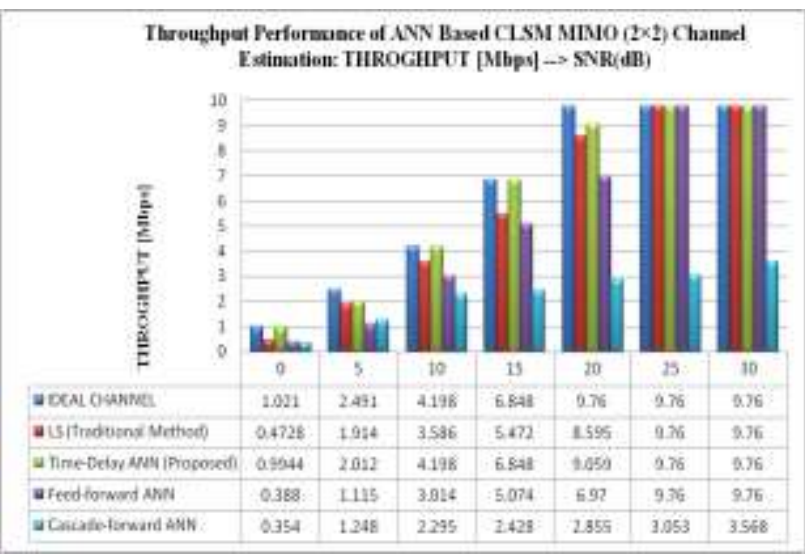

Fig. 5. Throughput v/s SNR performance for ANN based channel estimators in CLSM-MIMO $(2 \times 2)$ for AWGN Channel

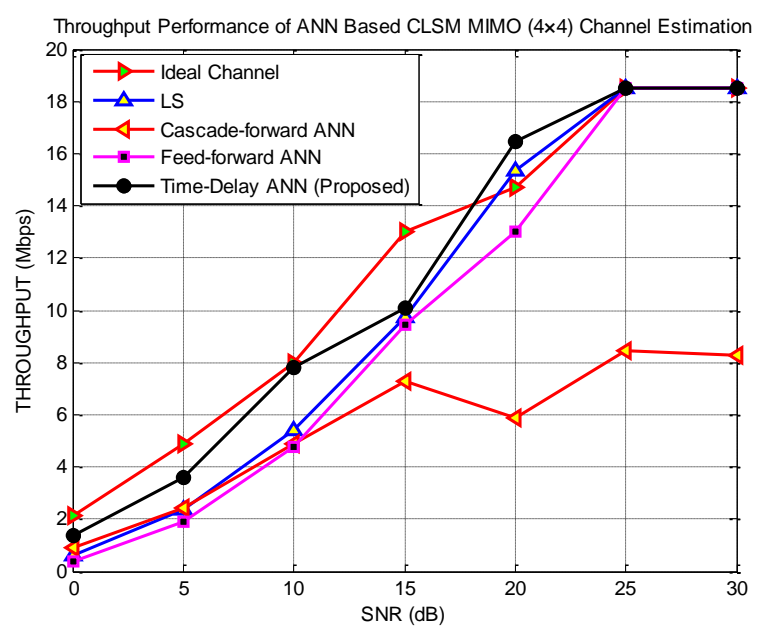

Fig. 6. Throughput v/s SNR performance for ANN based channel estimators in CLSM-MIMO $(4 \times 4)$ for AWGN Channel

From Fig. 4, 5, 6 and 7, Time delay neural networks (TDNN) gives 0.9944 Mbps, 2.012 Mbps, 4.198 Mbps, 6.848 Mbps, 9.059 Mbps, 9.76 Mbps and 9.76 Mbps for CLSM-MIMO $(2 \times 2)$ and also gives $1.349 \mathrm{Mbps}, 3.587$ Mbps, 7.828 Mbps, 10.07 Mbps, 16.46 Mbps, 18.51
Mbps and 18.51 Mbps at 0, 5, 10, 15, 20, 25 and $30 \mathrm{~dB}$ respectively for CLSM-MIMO $(4 \times 4)$ in AWGN channel which are the best performances when compared to Cascade-forward neural network, Feed-forward neural network and traditional Least Square (LS) methods. From Fig. 4 and 5, it is also giving throughput performance same as Ideal Channel for SNR range from $10 \mathrm{~dB}$ to 15 $\mathrm{dB}$ for CLSM-MIMO $(2 \times 2)$ in this channel.

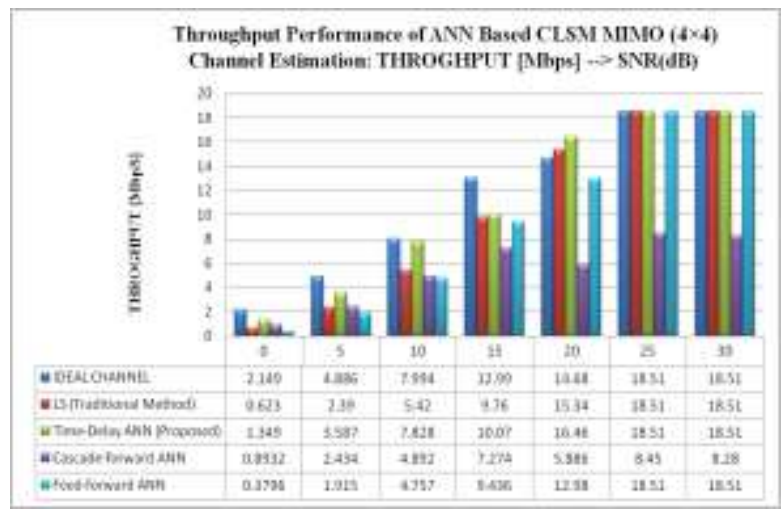

Fig. 7. Throughput v/s SNR performance for ANN based channel estimators in CLSM-MIMO $(4 \times 4)$ for AWGN Channel

Fig. 8, 9, 10, and 11 show the Throughput versus SNR results for different ANN based channel estimation method with LS channel estimator and Ideal Channel for Closed Loop Spatial Multiplexing (CLSM)-Single User Multi-input Multi-output (MIMO-2×2 and 4×4) for Flat Rayleigh Channel in LTE-A simulator. Fig. 9 and 10 are column chart representation of Fig. 8 and 10, respectively.

From Fig. 8, 9, 10 and 11, Cascade-forward neural network (CFNN) is the worst ANN for this application. Feed-forward neural network (FFNN) is giving better performance than Cascade-forward neural network. But, Time delay neural network (TDNN) is the most effective to improve performance and shows better results for SNR range $0 \mathrm{~dB}$ to $30 \mathrm{~dB}$ when compared to $\mathrm{ANN}$ based Channel estimators using FFNN and CFNN and traditional method LS estimator.

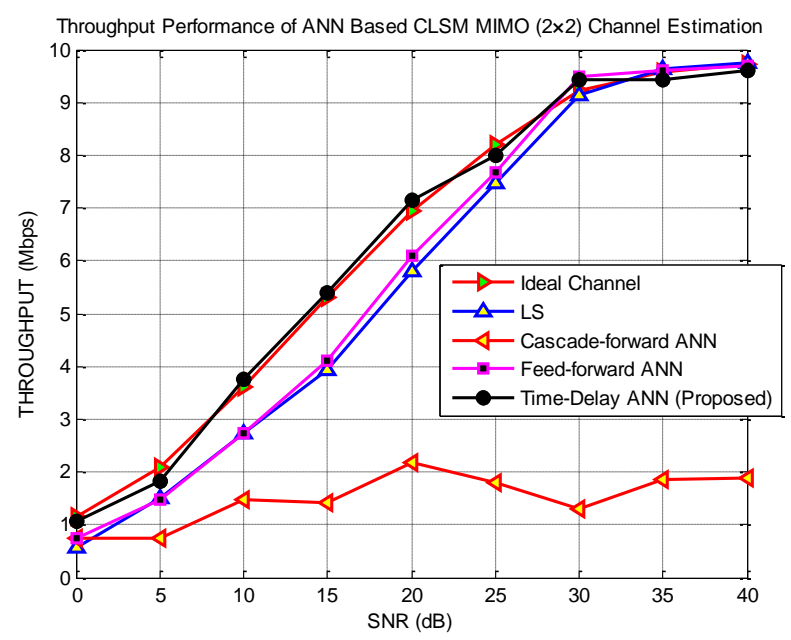

Fig. 8. Throughput v/s SNR performance for ANN based channel estimators in CLSM-MIMO $(2 \times 2)$ for Flat Rayleigh Channel 


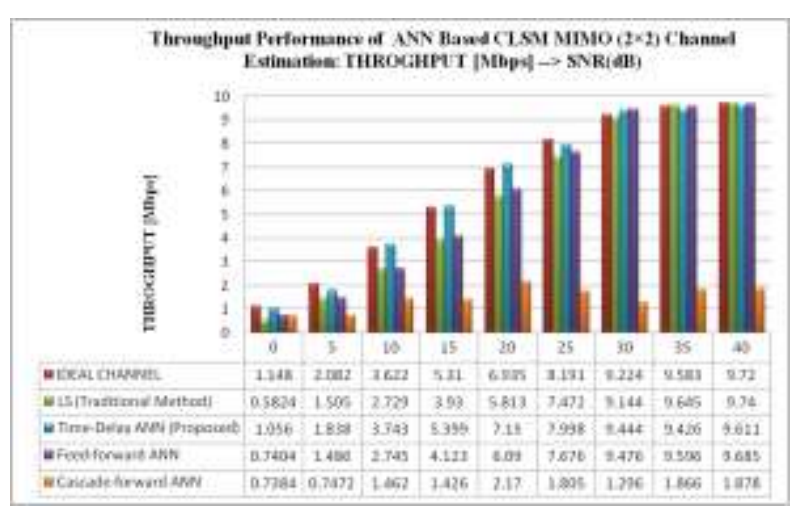

Fig. 9. Throughput v/s SNR performance for ANN based channel estimators in CLSM-MIMO $(2 \times 2)$ for Flat Rayleigh Channel

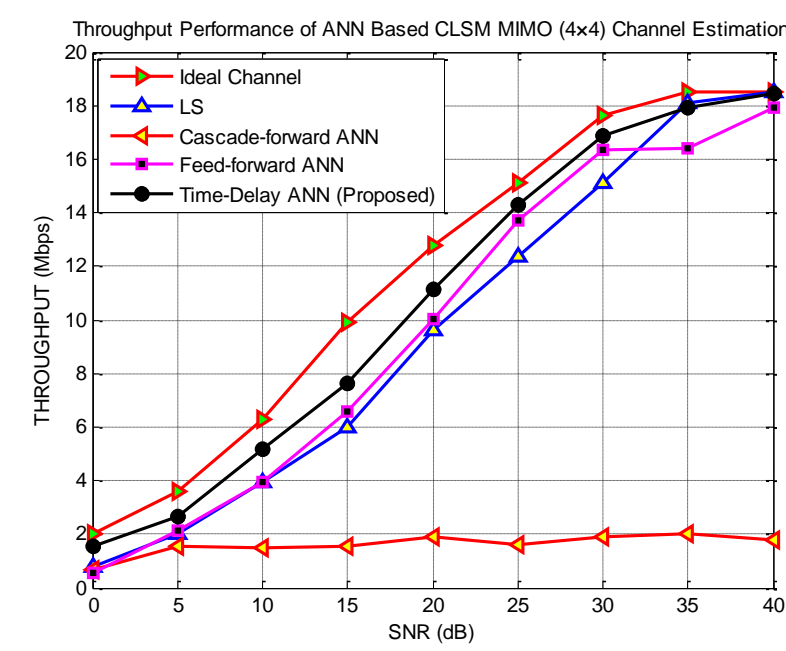

Fig. 10. Throughput v/s SNR performance for ANN based channel estimators in CLSM-MIMO $(4 \times 4)$ for Flat Rayleigh Channel

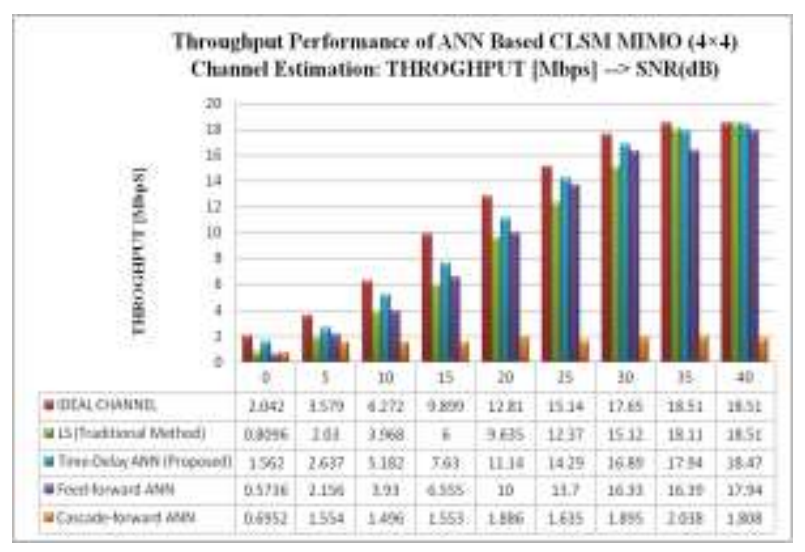

Fig. 11. Throughput v/s SNR performance for ANN based channel estimators in CLSM-MIMO $(4 \times 4)$ for Flat Rayleigh Channel

From Fig. 8, 9, 10 and 11, Time delay neural network (TDNN) gives 1.056 Mbps, $1.838 \mathrm{Mbps}, 3.743 \mathrm{Mbps}$, 5.399 Mbps, 7.15 Mbps, 7.998 Mbps and 9.444 Mbps for CLSM-MIMO $(2 \times 2)$ and 1.562 Mbps, 2.637 Mbps, 5.182 Mbps, 7.63 Mbps, 11.14 Mbps, 14.29 Mbps and 16.89 Mbps at 0, 5, 10, 15, 20, 25 and $30 \mathrm{~dB}$ respectively for CLSM-MIMO $(4 \times 4)$ in Flat Rayleigh Channel which are the best performances when compared to Feed-forward neural network, Cascade-forward neural network and traditional Least Square (LS) methods. But after SNR 30 $\mathrm{dB}$, TDNN is not much effective as in 0 to $30 \mathrm{~dB}$ SNR. From Fig. 8 and 9, it is also giving throughput performance better than Ideal Channel for SNR range from $10 \mathrm{~dB}$ to $20 \mathrm{~dB}$ for CLSM-MIMO $(2 \times 2)$ in this channel.

\section{CONCLUSION}

In this paper, Comparative Effect of Different Neural Networks on Throughput Performance of MIMO AWGN and Flat Rayleigh Channel Estimation for Downlink LTE-Advance System is introduced. This method is based on a learning process that uses a training sequence for adaptation to achieve a desired performance. Comparative throughput vs SNR analysis of proposed estimation method, Time delay neural network (TDNN) with traditional LS channel estimation technique and other different two ANN mechanism: FFNN and CFNN based channel estimators are carried out for Closed Loop Spatial Multiplexing-Single User Multi-input Multioutput $(2 \times 2$ and $4 \times 4)$ (CLSM-SUMIMO). MATLAB Simulation results show better performance in terms of throughput for the proposed TDNN. It shows better results when compared to traditional LS channel estimation technique and ANN based Channel estimation techniques using Cascade-forward neural network and Feed-forward neural network. Because, TDNN is similar to feed-forward networks, except that the input weight has a tap delay line associated with it. This allows the network to have a finite dynamic response to time series input data. The performance of channel estimator can be further enhanced by using Hybrid techniques for training Artificial Neural Networks like Genetic Algorithm train ANN, etc.

\section{ACKNOWLEDGMENT}

The author is grateful to the staff of Department of Electrical Engineering, Faculty of Technology and Engineering, The Maharaja Sayajirao University of Baroda and Electronics and Communication Engineering Department, Sarvajanik College of Engineering and Technology, Surat for their support. This work has been supported by the grant from Department of Science and Technology (DST), Government of India, under the Women Scientist (WOS-A) Scheme.

\section{REFERENCES}

[1] Armando Ubisse, Neco Ventura, "Modeling a Link Level Simulator for Long Term Evolution Uplink", Department of Electrical Engineering University of Cape Town, Communications Research Group Cape Town, South Africa, 2011.

[2] 3GPP TR 36.913, “3GPP; Technical Specification Group Radio Access Network. Requirements for further advancements for Evolved Universal 
Terrestrial Radio Access (E-UTRA)," v.9.0.0, December 2009.

[3] M. Rumney, "LTE and the Evolution to 4G Wireless: Design and Measurement Challenges", Agilent Technologies Publication, 2009.

[4] David Martin-Sacristan, Jose F. Monserrat, Jorge Cabrejas-Penuelas, Daniel Calabuig, Salvador Garrigas, and Narcis Cardona, "3GPP LTE and LTE-Advanced", January 2009.

[5] Jeanette Wannstrom, "LTE-Advanced", May 2012 http://www.3gpp.org/LTE-Advanced

[6] Nirmalkumar S. Reshamwala, Pooja S. Suratia, Satish K. Shah, "Study of ANN Configuration on Performance of Smart MIMO Channel Estimation for Downlink LTE-Advanced System", IJCNIS, vol.5, no.11, pp.27-35,2013. DOI: 10.5815/ijcnis.2013.11.04.

[7] A. Omri, R. Bouallegue, R. Hamila and M. Hasna, "Estimation of highly selective channels for downlink LTE MIMO-OFDM system by a robust neural network", International Journal of Wireless \& Mobile Networks (IJWMN), Vol.2, No. 1 (2011), pp. $31-38$.

[8] Barnali Dey, Awanish Kumar, Prashant Kumar, Sanjit Lal, Naveen Kumar, Bikash Sharma, "Channel Estimation using LS and MMSE Algorithm", International Symposium on Devices MEMS, Intelligent Systems \& Communication (ISDMISC) 2011.

[9] Saqib Saleem, "Channel Estimation using Adaptive Filtering for LTE-Advanced", International Journal of Computer Science (IJCSI) Issues, Vol. 8, Issue 3, No. 2, May 2011.

[10] Farooq Khan, "LTE for 4G Mobile Broadband Air Interface Technologies and Performance", Telecom R\&D Center Samsung Telecommunications, America, Cambridge University Press 2009.

[11] 3GPP TS 36.211, Evolved Universal Terrestrial Radio Access (EUTRA), "Physical Channels and Modulation", (Release 11).

[12] 3GPP TS 36.212, Evolved Universal Terrestrial Radio Access (EUTRA), "Multiplexing and channel coding", (Release 11).

[13] Satish K. Shah, Pooja S. Suratia, Nirmalkumar S. Reshamwala, "Comparative Performance Analysis of ANN Based MIMO Channel Estimation for downlink LTE- Advanced System employing Genetic Algorithm", proceeding of International Conference On Soft Computing and Software Engineering [SCSE'13].

[14] Nirmalkumar S. Reshamwala, Pooja S. Suratia, Satish K. Shah, "Performance Analysis of ANN Based MIMO Channel Estimation for downlink LTE-Advanced System", National Level Paper Contest called "Soft Computing for processing, Security Networking and Communication: (SCPSNC_2013), IETE Vadodara and Faculty of Technology, Electrical Engineering Department, M S University of Baroda, Vadodara, 20th January, 2013.
[15] [Online].Available:

http://www.nt.tuwien.ac.at/about-us/staff/josep-

colom-ikuno/lte-simulators/

[16] Albert Serra Pagès, "A Long Term Evolution Link Level Simulator", Universitat Politècnica de Catalunya February, 2009.

[17] LTE World, The Seven Modes of MIMO in LTE, [online]. Available: http://lteworld.org/whitepaper/seven-modes-mimolte.

[18] C. Mehlfuhrer, J. Colom Ikuno, M. Simko, S. Schwarz, M. Wrulich, M. Rupp, "The Vienna LTE Simulators - Enabling Reproducibility in Wireless Communications Research", EURASIP Journal on Advances in Signal Processing, Vol. 2011, pages 1 13,2011

[19] 3GPP Document TS 36.213, Evolved Universal Terrestrial Radio Access (EUTRA), "Physical Layer Procedure", (release 11).

[20] ITU-R M.1225 International Telecommunication Union, 'Guidelines for evaluation of radio transmission technologies for IMT-2000', 1997.

[21] Ericsson, Nokia, Motorola, and Rohde \& Schwarz, 'R4-070572: Proposal for LTE Channel Models', www. gpp.org, 3GPP TSG RAN WG4, meeting 43, Kobe, Japan, May 2007.

[22] Nirmalkumar S. Reshamwala, "Application of Soft Computing for Channel Estimation and Equalization in LTE”, Faculty of Technology and Engineering, The Maharaja Sayajirao University of Baroda, Vadodara, Gujarat, India, June-2013.

[23] Martin G. Reese, "Application of a time-delay neural network to promoter annotation in the Drosophila melanogaster genome", Computers and Chemistry $26 \quad\left(8^{\text {th }} \quad\right.$ May-2001) 51-56, www.elsevier.com/locate/compchem.

\section{Authors' Profiles}

Nirmalkumar S. Reshamwala was born in Surat, Gujarat, India, on May 10th, 1990. He has obtained B.E. (Electronics \& Communication) from Dharmsinh Desai University, Nadiad, Gujarat, India in May-2011 and has completed M.E. (Electrical Engineering-Automatic Control \& Robotics) in July-2013. He has published research papers in international conferences/Journals. Currently, he is a Assistant Professor in Electronics \& Communication department at Sarvajanik College of Engineering \& Technology, Surat, Gujarat, India. His areas of interests are Wireless Communication (LTE, 4G, etc), Networking, Channel Estimation and Equalization and SoftComputing.

Pooja S. Suratia is currently a Research Scholar in Department of Electrical Engineering, Faculty of Technology and Engineering, The Maharaja Sayajirao University of Baroda, Vadodara, Gujarat, India. She received Masters of Engineering Degree in Automatic Control and Robotics from The Maharaja Sayajirao University of Baroda (2009), Gujarat, India and a Bachelor of Engineering in Electronics and Communication from Sa'd Vidya Mandal Institute of Technology, Bharuch, Gujarat (2006), India. Her current research interests focus on 
MIMO Wireless Communication and Optimization of future wireless systems.

Prof. Satish Shah is a professor in the Electrical Engineering Department at Faculty of Technology, MS University of Baroda for last Twenty Five years. He is a fellow of IE(I) for past Fourteen Years and has also served as the member of Committee of Vadodara local center for more than Ten years in past. He has guided more than hundred projects at UG/PG level and completed a research project on DSP based Active Power filter sponsored by AICTE, New Delhi. He has written three books on Embedded System design / Microprocessors / Microcontrollers and presented/published more than 35 research papers in national / international conferences / Journals. He has attended and organized several seminars, workshops, and symposiums for UGC, AICTE, IETE, and MSU. He is a fellow of other technical associations such as: IETE, ISA and IEEE (NY) \& ISTE. He has served as the member, Hon Secretary and Treasurer of their local executive committees for a span of ten years.

How to cite this paper: Nirmalkumar S. Reshamwala, Pooja S. Suratia, Satish K. Shah,"Time-Delay Neural Network for Smart MIMO Channel Estimation in Downlink 4G-LTE-Advance System", International Journal of Information Technology and Computer Science(IJITCS), vol.6, no.6, pp.1-8, 2014. DOI: 10.5815/ijitcs.2014.06.01 